Article

\title{
Biological Features and Prognostic Impact of Bone Marrow Infiltration in Patients with Diffuse Large B-cell Lymphoma
}

\author{
Sara Alonso-Álvarez ${ }^{1,+}$, Miguel Alcoceba ${ }^{1,+}{ }^{+}$, María García-Álvarez ${ }^{1}$, Oscar Blanco ${ }^{2}$, \\ Marta Rodríguez ${ }^{2}$, Mónica Baile ${ }^{1}$, Juan Carlos Caballero ${ }^{1}$, Julio Dávila ${ }^{1}$, \\ María Belén Vidriales ${ }^{1}$, Carmen Esteban ${ }^{3}$, Piedad Arias ${ }^{4}$, Luis G. Díaz ${ }^{5}$, Pilar Tamayo ${ }^{5}$, \\ María Dolores Caballero ${ }^{1}$, Norma C. Gutiérrez ${ }^{1}$, Marcos González ${ }^{1}$ and Alejandro Martín ${ }^{1, *}$ \\ 1 Department of Hematology, University Hospital of Salamanca (HUS-IBSAL), CIBERONC, and Cancer \\ Research Institute of Salamanca-IBMCC (CSIC-USAL University), 37007 Salamanca, Spain \\ 2 Department of Pathology, University Hospital of Salamanca (HUS/IBSAL), 37007 Salamanca, Spain \\ 3 Department of General and Gastrointestinal Surgery, University Hospital of Salamanca (HUS/IBSAL), \\ 37007 Salamanca, Spain \\ 4 Department of Radiology, University Hospital of Salamanca (HUS/IBSAL), 37007 Salamanca, Spain \\ 5 Department of Nuclear Medicine, University Hospital of Salamanca (HUS/IBSAL), 37007 Salamanca, Spain \\ * Correspondence: amartingar@usal.es \\ + S.A.-Á. and M.A. contributed equally to this study.
}

Received: 16 January 2020; Accepted: 14 February 2020; Published: 18 February 2020

check for updates

\begin{abstract}
The biology and clinical impact of bone marrow (BM) infiltration in patients with diffuse large B-cell lymphoma (DLBCL) remains unclear in the rituximab era. We retrospectively analyzed 232 patients diagnosed with DLBCL at our center between 1999 and 2014. Concordant-presence of large cells similar to those of the lymph node biopsy- and discordant-infiltration by small cells forming lymphoid aggregates, lacking cytological atypia-BM infiltration was defined by histological criteria and further characterized by flow cytometry (FCM). Cell of origin (COO) was determined using Hans' algorithm. For the clonal relationship between tumor and discordant BM, the VDJH rearrangement was analyzed. Survival analyses were restricted to 189 patients treated with rituximab and chemotherapy. Thirty-six (16\%) had concordant, and 37 (16\%) discordant BM infiltration. FCM described different indolent lymphomas among discordant cases, clonally related with DLBCL in 10/13 available samples. Median follow-up was 58 months. 5-year-progression-free survival (PFS) for non-infiltrated, discordant and concordant groups was 68\%, 65\% and 30\%, respectively $(p<0.001)$. Combining COO and BM infiltration, patients with discordant BM and non-germinal center B-cell COO also had decreased 5-year-PFS (41.9\%). In multivariate analysis, concordant BM had an independent effect on PFS (HR 2.5, $p=0.01$ ). Five-year cumulative incidence of central nervous system (CNS) relapse was $21 \%, 4 \%$ and $1 \%$ in concordant, discordant and non-infiltrated groups, respectively $(p<0.001)$. In conclusion, concordant BM infiltration represents a subset with poor prognosis, whereas the prognostic impact of discordant BM infiltration could be limited to non-CGB cases.
\end{abstract}

Keywords: Bone marrow involvement; Diffuse large B-cell lymphoma; Discordant bone marrow; Concordant bone marrow; CNS relapse

\section{Introduction}

Bone marrow (BM) is involved in 11-25\% of patients diagnosed with diffuse large B-cell lymphoma (DLBCL) [1-4]. It is well known that this involvement is not always concordant with the aggressive 
lymphoma histology observed in the diagnostic tumor sample [5]. Several studies indicate that low-grade histology in BM (discordant BM) is present in $30-50 \%$ of DLBCL patients with BM infiltration [1-4]. Previous work has shown the unfavorable prognosis determined by a concordant marrow infiltration, with lower progression free survival (PFS) and overall survival (OS) $[1-4,6]$. The prognostic role of discordant BM infiltration is unclear, but it seems to be less unfavorable than concordant involvement, as no study has proven that discordant $\mathrm{BM}$ is an independent prognostic factor for OS. In addition, the role of BM involvement as a risk factor for central nervous system (CNS) relapse is controversial in the rituximab era $[7,8]$.

Some studies have incorporated cytomorphological, immunophenotypic and molecular analysis, including copy number variation analysis, to improve the sensitivity of the tumor infiltrate detection, and to provide a detailed biological description $[1,9,10]$. However, little information is available regarding the biological mechanisms involved in the development of both types of infiltrate. Furthermore, it is not known whether there is any relation between the type of infiltration and the cell of origin of DLBCL. In addition, the possible clonal relationship between the tumor and BM clones in discordant cases has barely been studied [9]. With the little genetic data available, we cannot confirm whether the relation between low-grade and high-grade histologies is similar to what has been described in other transformation models.

On this basis, we retrospectively analyzed our series of patients diagnosed with DLBCL to examine the prognostic impact and biological features of discordant and concordant BM infiltration. We included flow cytometry (FCM) analysis of the diagnostic BM aspirates, to improve the sensitivity of tumor detection and to characterize phenotypically low-grade infiltrates. We also questioned the relationship of the cell of origin (COO) of the aggressive tumor sample and the type of BM infiltration. In addition, we analyzed the possible clonal relationship between the tumor and BM clones in discordant cases.

\section{Patients and Methods}

\subsection{Study Design and Patients}

This is a retrospective observational study performed in the Hospital Universitario de Salamanca. We identified patients by reviewing Hematology and Pathology Department databases, and selecting those cases with a histological diagnosis of DLBCL. We included all consecutively diagnosed patients between 1999 and 2014 with staging BM biopsy. Those with DLBCL transformed from previous indolent lymphoma and primary central nervous system lymphomas were excluded. The study was approved by our institutional ethics committee (code: "PI 201611 001").

\subsection{Histopathological Review and Immunohistochemistry}

An expert hemopathologist reviewed all of the BM core biopsies for the present study. Concordant and discordant BM infiltration was defined by histological criteria. Concordant involvement was defined as the presence of large cells, similar to those present in the initial lymph node diagnostic biopsy. Discordant involvement was defined as infiltration by small cells forming lymphoid aggregates, lacking cytological atypia. In some cases, the pathologist reported an unspecific lymphoid proliferation not suggestive of malignancy; in those cases, flow cytometry (FCM) was used to characterize the infiltrates further, and whether a clonal B-cell population of any size was found by FCM, these were included in the discordant group.

The cell of origin (COO) was determined in the original tumor sample using immunohistochemistry (IHC), according to the algorithm of Hans et al [11]. DLBCL cases were classified as germinal center B-cell-like (GCB) or non-GCB. 


\subsection{Flow Cytometry Analysis}

BM aspirates obtained in the same procedure as the BM biopsies were analyzed by FCM, and when available, were sent to the Molecular Biology and Cytogenetic Laboratories as part of the routine extension study for DLBCL.

BM aspirates were processed according to conventional procedures [12,13]. Samples were stained using a four-color (from 1999 to 2010) or eight-color (from 2011 to 2014) direct immunofluorescence technique. For four-color protocols, the following monoclonal antibody $(\mathrm{MoAb})$ combinations (FITC/PE/PerCP-Cy5.5/APC) were used for screening: FMC7/CD5/CD19/CD45, and sK/sL/CD19/CD20. For eight-color protocols, the MoAb combinations (FITC/PE/PerCP-Cy5.5/PE-Cy7/ APC/APC-H7/BV450/OC515) used were: sK/sL/CD5/CD19/CD10/CD38/CD20/CD45.

After staining, samples were acquired on a FACSCalibur or FACSCanto-II flow cytometer (Becton/Dickinson Biosciences (BD), San José, CA, USA), using CellQUEST or DIVA software (BD), respectively. For the analysis, the Paint-a-Gate (Becton Dickinson Biosciences) or INFINICYT ${ }^{\mathrm{TM}}$ software programs (Cytognos SL, Salamanca, Spain) were used.

\subsection{Clonality Analysis and IGHV Gene Sequencing}

Tumor DNA was extracted from samples collected at the time of diagnosis. In fresh samples, high-molecular-weight DNA was isolated using DNAzol reagent (MRC, Cincinnati, OH, USA). Clonality assessment and immunoglobulin heavy-chain variable region (IGHV) genes rearrangement were analyzed in both lymph node and bone marrow samples from the group of patients with discordant BM involvement (histologically and/or phenotypically documented). Tumor samples were tested for the amplification of IGH rearrangements according to the BIOMED-2 Concerted Action protocols [14]. Complete V-D-J rearrangement amplification was performed by multiplex PCR with a set of family-specific primers of the framework region 1 (FR1) and one IGHJ consensus primer. For the samples with no detectable amplification from FR1, PCR was performed with the FR2 region. The presence of the monoclonal rearrangement was confirmed by GeneScan with using ABI 3130xL or ABI 3500xL DNA Sequencers (Applied Biosystems, Foster City, California). PCR products were then sequenced directly, using Big-Dye terminators (Applied Biosystems). Germline IGHV genes from complete V-D-J rearrangements were identified using the IMGT/V-QUEST database (http://www.imgt.org, last accessed November 18 2016). We recorded IGHV gene usage, and the percentage of IGHV identity to the closest germline gene.

\subsection{Fluorescence in Situ Hybridization Analysis}

Interphase fluorescence in-situ hybridization (FISH) studies were carried out in the group of DLBCL with involved bone marrow. Carnoy fixed cells preserved at diagnosis of lymph node samples were used in most patients, although in those concordant cases without lymph node available, the infiltrated BMs were used. MYC and BCL6 rearrangements, $\mathrm{t}(14 ; 18)(\mathrm{q} 32 ; \mathrm{q} 21)(B C L 2)$ and 17p13.1 region (TP53) were explored by using the FISH probes, "LSI MYC and LSI BCL6 Dual Color Break Apart Rearrangement", "IGH/BCL2 Dual Color Dual Fusion Translocation" and "LSI TP53" acquired from Abbott Molecular (Des Plaines, IL) following the manufacturer's recommendations.

\subsection{Endpoints and Statistical Methods}

Primary endpoints were i) PFS, calculated from the date of diagnosis until the date of relapse, progression, death from any cause, or last follow-up; ii) cumulative incidence of CNS relapse, documented by FCM or image techniques (computed tomography and/or magnetic resonance imaging) when clinically suggestive; and iii) OS, calculated from the date of diagnosis until the date of death from any cause or last follow-up.

Survival analyses was only performed among the group of patients $(n=189)$ who had been homogeneously treated with rituximab plus chemotherapy with a curative intent. Clinical data were 
last updated on November 18 2016. The main clinical-biological variables at diagnosis were abstracted, including the date of birth, date of DLBCL diagnosis, revised International Prognostic Index (R-IPI) [15] variables and score, the involvement of other extranodal sites, CNS-IPI score [16], first-line therapy, progression, death and date of last follow-up. All patients were staged at diagnosis with computed tomography and bone marrow biopsy.

We considered death as a competing risk to calculate the cumulative incidence of CNS relapse. Cumulative incidence curves were plotted, and Gray's test was used to calculate the statistical differences, using R software (available at www.r-project.org). Survival curves were plotted using the Kaplan-Meier method, and the differences were analyzed using the log-rank or Breslow test, as appropriate. Multivariate analysis was carried out using the time-dependent Cox-proportional hazard model, including all variables with some indication of a significant association in the univariate test $(p<0.1)$ or those considered relevant in previous reports. Group means were compared using Student's t-test. Qualitative variables were analyzed by the Pearson $\chi^{2}$ test or Fisher's exact test, as appropriate. Two-tailed values of $p<0.05$ were considered significant. All analyses were carried out with SPSS, v.20 (IBM, Armonk, NY, USA).

\section{Results}

\subsection{The Combination of Histology and Flow Cytometry Improved the Bone Marrow Involvement Classification}

A total of 232 patients sequentially diagnosed with DLBCL in our center between January 1 1999 and December 312014 were included. According to their histology, 57 patients (25\%) had BM infiltration, of which $36(16 \%)$ were concordant, and 21 (9\%) discordant. FCM analysis revealed lymphoma infiltration in $16(7 \%)$ additional patients with unspecific lymphoid infiltrates, and were included in the discordant group. Clinical characteristics of patients are summarized in Table 1. As shown in Table 1, poor-risk (R-IPI) was more frequent among patients in the concordant group $(85 \%)$ than in the discordant $(59 \%)$ or non-infiltrated $(41 \%)$ groups $(p<0.001)$.

Table 1. Patient characteristics according to the bone marrow infiltration.

\begin{tabular}{|c|c|c|c|c|}
\hline Variable & $\begin{array}{l}\text { Non-infiltrated BM } \\
\quad(n=159)\end{array}$ & $\begin{array}{c}\text { Discordant BM } \\
\quad(n=37)\end{array}$ & $\begin{array}{c}\text { Concordant BM } \\
(n=36)\end{array}$ & $p$ \\
\hline Gender (Male) & $82(52 \%)$ & $12(32 \%)$ & $20(56 \%)$ & 0.07 \\
\hline Age $>60$ years & $106(67 \%)$ & $27(73 \%)$ & $20(56 \%)$ & 0.3 \\
\hline Ann Arbor stage $>2$ & $91(58 \%)$ & $37(100 \%)$ & $36(100 \%)$ & $<0.001$ \\
\hline LDH (high) * & $50(39 \%)$ & $13(41 \%)$ & $20(77 \%)$ & 0.0018 \\
\hline Extranodal sites $>1 *$ & $27(20 \%)$ & $16(48 \%)$ & $19(68 \%)$ & $<0.001$ \\
\hline $\mathrm{ECOG} \geq 2 *$ & $41(32 \%)$ & $8(25 \%)$ & $14(54 \%)$ & 0.05 \\
\hline R-IPI > 2 * & $54(41 \%)$ & $20(59 \%)$ & $22(85 \%)$ & $<0.001$ \\
\hline COO non-GCB * & $64(63 \%)$ & $19(62 \%)$ & $19(64 \%)$ & 0.99 \\
\hline
\end{tabular}

${ }^{*}$ In the descriptive analysis, either ECOG, R-IPI, LDH or extranodal sites data was not available in 32 non-infiltrated $\mathrm{BM}, 5$ discordant $\mathrm{BM}$, and 10 concordant $\mathrm{BM}$; $\mathrm{COO}$ was not available in 58,7 and 6 cases, respectively. Regarding patients included in the survival analysis $(\mathrm{n}=189)$, either ECOG, R-IPI, LDH or extranodal sites data were not available in 9/134 patients with non-infiltrated BM, 2/26 with discordant BM and 1/29 with concordant BM, while $\mathrm{COO}$ was not available in 33, 3 and 2 cases, respectively. Abbreviations: BM: bone marrow; COO: Cell-of-origin; R-IPI: revised International Prognostic Index.

FCM results in BM were available in 35 out of 36 patients with concordant BM infiltration, and all 21 patients with discordant BM infiltration (Table 2). In concordant cases, FCM results were suggestive of DLBCL in most patients. In contrast, FCM showed a wide variety of indolent B-cell lymphomas in the discordant cases (Table 2). FCM did not show tumor infiltration in 14 cases with discordant $(n=4)$ or concordant $(n=10)$ histological BM infiltration, probably due to the type of infiltration, which, in all cases, was either focal or interstitial. 
We found no statistically significant associations between the cell of origin of the DLBCL and the type of BM infiltration. Thus, $64 \%$ of concordant cases were non-GCB-DLBCL while $62 \%$ of discordant cases, and $63 \%$ in the non-infiltrated BM group $(p=1)$.

Table 2. Phenotypic characteristics of bone marrow (BM) lymphoid infiltrates according to histology.

\begin{tabular}{ccccccccc}
\hline Histology & \multicolumn{7}{c}{ FCM } \\
\cline { 2 - 9 } & DLBCL & DLBCL+FL & FL & CLL & MZL-like & LPL-like & NS & Non-Infiltrated \\
\hline $\begin{array}{c}\text { High-grade (Concordant } \\
\text { BM, n = 35) }\end{array}$ & 21 & - & 3 & - & - & - & 1 & 10 \\
\hline $\begin{array}{c}\text { Low-grade (Discordant } \\
\text { BM, n = 21) }\end{array}$ & 1 & 1 & 3 & 4 & 2 & - & 6 & 4 \\
\hline $\begin{array}{c}\text { Unspecified lymphoid } \\
\text { infiltrate (n = 31)* }\end{array}$ & 1 & - & 4 & 4 & 2 & 1 & 4 & 15
\end{tabular}

* The 16 cases with a clonal B-cell population of any size found by FCM were included in the discordant group. Abbreviations: CLL: chronic lymphocytic leukemia; DLBCL: diffuse large B-cell lymphoma; FL: follicular lymphoma; MZL: marginal zone lymphoma. LPL: lymphoplasmacytic lymphoma. NS: B-cell NHL with non-specific phenotype.

\subsection{Clonality Studies Revealed a Unique Clone in most of the Discordant Cases}

We had 18 paired lymph node and BM samples from the group of discordant patients (histologically and/or phenotypically documented). A polyclonal trace was observed in at least one sample in five cases, and therefore the clonality relationship was undetermined. A clonal peak could be identified in 13 cases, which revealed a common monoclonal peak in 10 patients, and different clones in three patients, all of them with a CLL phenotype. We sequenced IGHV genes in those patients with a common clonal origin. The genes most frequently used were IGHV4-34 $(\mathrm{n}=2)$ and IGHV3-48 $(\mathrm{n}=2)$, and there were six individual cases for IGHV1-3, IGHV1-8, IGHV3-7, IGHV3-33, IGHV3-53 and IGHV3-72, respectively.

\subsection{FISH Analysis Did Not Find Different Genetic Abnormalities between Concordant and Discordant Groups}

Twenty-one patients, 14 with concordant BM infiltration and 7 with discordant infiltration, could be analyzed by FISH (Table 3). Most of the patients with concordant and discordant BM showed genetic aberrations in one or more of the analyzed genes (64\% in concordant $\mathrm{BM}$, and $86 \%$ in discordant BM group, $p=\mathrm{NS}$ ). Interestingly, discordant BM cases showed gains in MYC more frequently than concordant BM $(57 \%$ vs. $14 \%, p=0.06)$. No other genetic alteration differs between BM involvement groups.

$B C L 2$ alterations were differentially distributed between COO groups. Thus, BCL2 translocations were more frequently found in GCB-DLBCL (70\% in GCB-DLBCL vs. 9\% in non-GCB, $p=0.007$ ), while BCL2 gains were only found in non-GCB-DLBCL ( $54 \%$ vs. $0 \%, p<0.001)$, independently of the type of BM infiltration. Double-hit translocations affecting BCL2 and MYC $(\mathrm{n}=2)$ and triple-hit translocations affecting BCL2, MYC and BCL6 ( $\mathrm{n}=1)$ were only observed in the GCB group. Interestingly, one patient showed a double hit translocation (BCL2 and MYC), and was the only one with discordant BM and GCB COO that progressed after rituximab plus curative chemotherapy.

Table 3. Fluorescence in-situ hybridization (FISH) analysis of patients with involved bone marrow.

\begin{tabular}{ccccccccc}
\hline UPN & Sample & \% FCM & $\begin{array}{c}\mathbf{( 1 4 ; 1 8 )} \\
(\text { IGH/BCL2) }\end{array}$ & $\begin{array}{c}\mathbf{8 q 2 4} \\
(\boldsymbol{M Y C})\end{array}$ & $\begin{array}{c}\mathbf{3 q 2 6} \\
\mathbf{( B C L 6 )}\end{array}$ & $\begin{array}{c}\mathbf{1 7 p 1 3 . 1} \\
\mathbf{( T P 5 3 )}\end{array}$ & COO & BM \\
\hline 3470 & LN & 60 & Clonal & Clonal & Clonal & Normal & GCB & C \\
7062 & LN & 9,5 & Clonal & Normal & Normal & Normal & GCB & C \\
4512 & BM & 21 & Normal & Normal & Normal & Normal & GCB & C \\
3999 & LN & 12 & Normal & Normal & Normal & Normal & GCB & C \\
9102 & BM & 15 & Clonal & Normal & Gain & Normal & GCB & C \\
9225 & LN & 66,7 & Clonal & Clonal & Normal & Normal & GCB & C \\
2704 & BM & 15 & Gain & Normal & Normal & Normal & Non-GCB & C \\
\hline
\end{tabular}


Table 3. Cont.

\begin{tabular}{|c|c|c|c|c|c|c|c|c|}
\hline UPN & Sample & $\%$ FCM & $\begin{array}{c}(14 ; 18) \\
(I G H / B C L 2)\end{array}$ & $\begin{array}{c}8 \mathrm{q} 24 \\
(M Y C)\end{array}$ & $\begin{array}{c}3 \mathrm{q} 26 \\
(B C L 6)\end{array}$ & $\begin{array}{c}\text { 17p13.1 } \\
(T P 53)\end{array}$ & $\mathrm{COO}$ & BM \\
\hline $3985^{*}$ & LN & 47 & Gain & Gain & Gain & Gain & Non-GCB & $\mathrm{C}$ \\
\hline 6908 & LN & 15 & Normal & Normal & Normal & Normal & Non-GCB & $\mathrm{C}$ \\
\hline $\begin{array}{c}6156 \\
+\end{array}$ & LN & $N A$ & Gain & Gain & Gain & Gain & Non-GCB & $\mathrm{C}$ \\
\hline 7288 & LN & 69 & Gain & Normal & NS & Normal & Non-GCB & $\mathrm{C}$ \\
\hline 5296 & LN & 15 & Normal & Normal & Normal & Normal & Non-GCB & $\mathrm{C}$ \\
\hline 8275 & BM & 36,6 & Normal & Normal & Normal & Normal & Non-GCB & $\mathrm{C}$ \\
\hline 9043 & LN-FFPE & $N A$ & Gain & Clonal & Normal & NS & Non-GCB & $\mathrm{C}$ \\
\hline 4859 & LN & $N A$ & Clonal & Gain & Normal & Normal & GCB & $\mathrm{D}$ \\
\hline 4989 & LN & 75 & Clonal & Normal & Clonal & Normal & GCB & $\mathrm{D}$ \\
\hline 7029 & $\mathrm{LN}$ & 78 & Normal & Gain & Gain & Normal & GCB & $\mathrm{D}$ \\
\hline $8724^{*}$ & LN & 93 & Clonal & Clonal & Gain & Gain & GCB & $\mathrm{D}$ \\
\hline 3671 & LN & 60 & Normal & Normal & Normal & Normal & Non-GCB & $\mathrm{D}$ \\
\hline 8818 & LN & 48 & Clonal & Gain & Normal & Normal & Non-GCB & $\mathrm{D}$ \\
\hline 9386 & LN & 64 & Gain & Gain & Gain & Deletion & Non-GCB & $\mathrm{D}$ \\
\hline
\end{tabular}

* Tetraploid karyotype; + Hyperdiploid karyotype. Abbreviations: BM: bone marrow; C: concordant infiltration; COO: cell of origin; D: discordant infiltration; FCM: Flow cytometry; FFPE: formalin-fixed paraffin-embedded; GCB: germinal center B-cell like; LN: lymph node; NA: not available.

\subsection{Concordant BM Cases Displayed Shorter PFS and Higher Incidence of CNS Relapse}

Survival was only analyzed in the 189 patients treated with rituximab plus chemotherapy with curative intent (R-CHOP [rituximab, cyclophosphamide, doxorubicin, vincristine, prednisone] $\mathrm{n}=152$ $(80.4 \%)$, R-MegaCHOP $n=22(11.6 \%)$, R-COMP [pegylated doxorubicin instead doxorubicin] $n=5(2.6 \%)$, VR-CAP [bortezomib instead vincristine] $n=4(2.1 \%)$ and others $n=6(3.2 \%))$. Nine of 31 patients $(29 \%)$ in the discordant $\mathrm{BM}$ group and 13 of 35 patients $(37 \%)$ in the concordant group received prophylaxis for CNS relapse, consisting of 4-6 doses of triple intrathecal therapy (methotrexate $12 \mathrm{mg}$, cytarabine $30 \mathrm{mg}$ and hydrocortisone $20 \mathrm{mg}$ ). Median follow-up of surviving patients was 58 months (1-152 months).

PFS at 5 years was $68 \%$ for the non-infiltrated group, $62 \%$ for the discordant group $(p=0.4)$ and $32 \%$ for the concordant group $(p<0.001)$ (Figure $1 \mathrm{~A})$. In the multivariate analysis including COO and R-IPI, concordant BM infiltration had an independent impact on PFS, over and above that of the R-IPI as a global score $(\mathrm{HR}=2.2 ; 95 \% \mathrm{CI}=1.1-4.3 ; p=0.02)$ (Table 4$)$, when categorized according to risk groups, or when testing the individual variable content in the R-IPI separately (results not shown).

Patients with concordant BM had a lower 5-year OS (51\%) than those in the discordant $(72.5 \%)$ and non-infiltrated $(72.5 \%)$ groups $(p=0.06)$ (Figure $1 \mathrm{~B})$, although the only variable with independent impact on OS was the R-IPI (Table 4). In the concordant group, 6 out of 18 patients with progressive disease received autologous stem-cell transplantation as salvage therapy, and two of them needed to be rescued afterwards with allogeneic stem-cell transplantation (allo-SCT). Four of them, including both who received allo-SCT, were disease-free at the time of writing.

Table 4. Multivariate analysis of progression-free survival and overall survival.

\begin{tabular}{ccccccc}
\hline \multirow{2}{*}{ Variable } & \multicolumn{3}{c}{ PFS } & \multicolumn{3}{c}{ OS } \\
\cline { 2 - 7 } & HR & $\mathbf{9 5 \%} \mathbf{C I}$ & $p$-Value & HR & $\mathbf{9 5 \%}$ CI & $p$-Value \\
\hline Bone marrow infiltration & - & - & - & - & - & - \\
No infiltration (reference) & - & - & - & - & - & - \\
Concordant & 2.2 & $1.1-4.3$ & 0.02 & 1.6 & $0.7-3.4$ & 0.2 \\
Discordant & 1.5 & $0.7-3$ & 0.2 & 1.5 & $0.7-3.2$ & 0.3 \\
R-IPI score (0 to 5) & 1.3 & $1.0-1.5$ & 0.03 & 1.4 & $1.1-1.8$ & 0.004 \\
\hline
\end{tabular}


Table 4. Cont.

\begin{tabular}{ccccccc}
\hline \multirow{2}{*}{ Variable } & \multicolumn{3}{c}{ PFS } & \multicolumn{3}{c}{ OS } \\
\cline { 2 - 7 } & HR & $\mathbf{9 5 \%}$ CI & $p$-Value & HR & $\mathbf{9 5 \% ~ C I ~}$ & $p$-Value \\
\hline Bone marrow infiltration and COO & & & & & & - \\
No infiltration (reference) & - & - & - & - & - & 0.2 \\
Concordant GCB & 2.9 & $1-8.7$ & 0.04 & 1.2 & $0.3-4.4$ & 0.3 \\
Concordant Non-GCB & 3 & $1.4-6.4$ & 0.003 & 1.6 & $0.7-3.9$ & 0.7 \\
Discordant GCB & 0.7 & $0.1-3$ & 0.6 & 0.7 & $0.1-3.4$ & 0.7 \\
Discordant Non-GCB & 1.9 & $0.9-4.2$ & 0.08 & 1.6 & $0.6-3.7$ & 0.3 \\
R-IPI score (0 to 5) & 1.2 & $0.9-1.5$ & 0.1 & 1.5 & $1.2-1.9$ & $<0.001$ \\
\hline
\end{tabular}

Abbreviations: COO: Cell of origin; GCB: germinal center B-cell like; R-IPI: revised International Prognostic Index; OS: overall survival; PFS: progression-free survival.

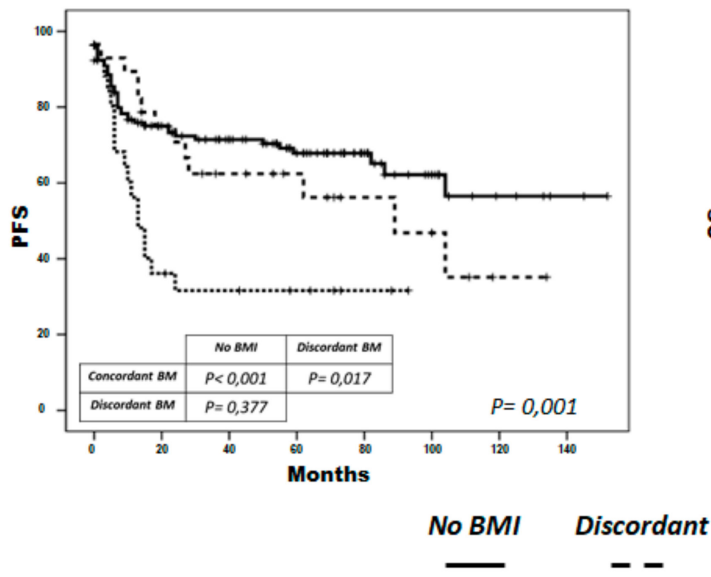

(A)

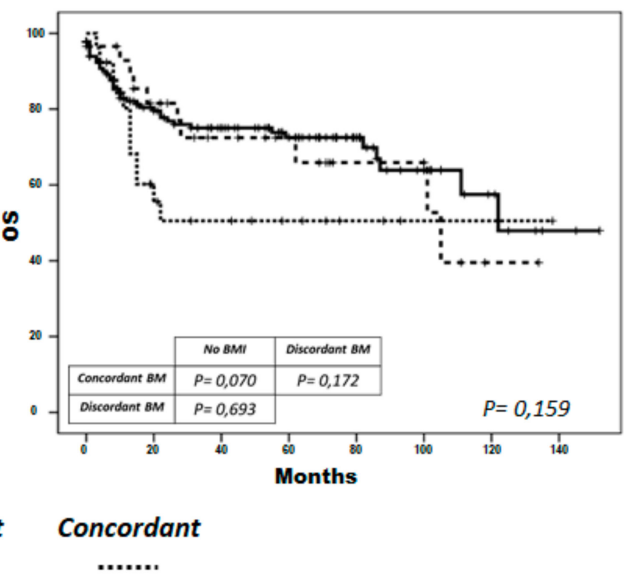

(B)

Figure 1. Progression-free survival (PFS) (A) and overall survival (OS) (B) by bone-marrow infiltration type. PFS and OS were significantly lower $(p<0.001$ and $p=0.08$, respectively) in patients with concordant infiltration (dotted line), than in those without BM infiltration (continuous line) and with discordant infiltration (discontinuous line). No significant differences were observed between the latter two groups $(\mathrm{p}>0.1)$.

We have reanalyzed PFS and OS, only considering those patients with stage IV in order to assess the prognostic impact of BM infiltration in comparison with other extranodal sites. In this setting, PFS at 5 years was $63 \%$ for the non-infiltrated group, $62 \%$ for the discordant group and $32 \%$ for the concordant group ( $p<0.05$, Figure S1A). There were no statistically significant differences in terms of 5 -year OS in concordant BM (51\%) as compared to discordant (72.5\%) and non-infiltrated (63\%) groups $(p=0.5)$ (Figure S1B).

Five-year cumulative incidence of CNS relapse was significantly higher in the concordant group (21\%) as compared to the discordant group (4\%) and the non-infiltrated group $(1 \%, p<0.001)$ (Figure 2$)$. In the multivariate analysis, including the type of BM infiltration and the variables included in CNS-IPI, concordant infiltration had an independent association with CNS relapse (HR: 10.1; 95\% CI: 2.2-46.3; $p=0.003$ ) (Table 5). 
Table 5. Univariate and multivariate analysis of central nervous system relapse incidence.

\begin{tabular}{|c|c|c|c|c|c|}
\hline \multirow{2}{*}{ Variable } & \multicolumn{2}{|l|}{ Univariate } & \multicolumn{3}{|c|}{ Multivariate } \\
\hline & 5-Year CNS Incidence & $p$-Value & $p$-Value & HR & $95 \% \mathrm{CI}$ \\
\hline $\begin{array}{c}\text { Bone marrow infiltration } \\
\text { No infiltration } \\
\text { Discordant } \\
\text { Concordant }\end{array}$ & $\begin{array}{l}0.8 \% \\
7.3 \% \\
3.1 \%\end{array}$ & $<0.001$ & 0.003 & 10.1 & $2.2-46.3$ \\
\hline $\begin{array}{c}\text { Renal/Adrenal } \\
\text { involvement } \\
\text { No } \\
\text { Yes }\end{array}$ & $\begin{array}{c}3.8 \% \\
22.2 \%\end{array}$ & 0.007 & 0.017 & 7.8 & $1.5-42$ \\
\hline $\begin{array}{c}\text { Lactate dehydrogenase } \\
\text { No } \\
\text { Yes }\end{array}$ & $\begin{array}{l}3.5 \% \\
13 \%\end{array}$ & 0.3 & - & - & - \\
\hline $\begin{array}{c}\text { ECOG } \\
0-1 \\
2-4\end{array}$ & $\begin{array}{l}4.2 \% \\
10 \%\end{array}$ & 0.8 & - & - & - \\
\hline $\begin{array}{l}\text { Ann Arbor stage } \\
\quad<3 \\
\geq 3\end{array}$ & $\begin{array}{l}8 \% \\
7 \%\end{array}$ & 0.4 & - & - & - \\
\hline $\begin{array}{c}\text { Extranodal sites } \\
<2 \\
\geq 2\end{array}$ & $\begin{array}{c}3.7 \% \\
12.5 \%\end{array}$ & 0.1 & - & - & - \\
\hline $\begin{array}{l}\text { Cell of origin } \\
\text { GCB } \\
\text { Non-GCB }\end{array}$ & $\begin{array}{l}6.8 \% \\
3.5 \%\end{array}$ & 0.8 & - & - & - \\
\hline
\end{tabular}

Abbreviations: CNS: central nervous system; GCB: germinal center B-cell like.

\subsection{Non-GCB discordant BM Cases Could Display Shorter PFS}

By combining the type of BM infiltration and COO, we observed that both concordant non-GCB $(n=16)$ and GCB $(n=8)$ patients had a lower 5-year PFS (33\% and 25\%, respectively) than the non-infiltrated GCB $(\mathrm{n}=32)$ and non-GCB $(\mathrm{n}=64)$ groups $(78.5 \%$ and $70 \%$, respectively), and the discordant GCB $(\mathrm{n}=9)$ group $(76 \%)$ ( $p<0.001$ for all comparisons). Notably, 5-year PFS in the discordant non-GCB group $(n=17)$ was $46 \%$, a significantly worse figure than in the non-infiltrated group (Figure 3A). In the multivariate analysis, concordant GCB and non-GCB groups had a higher risk of progression independently of R-IPI; the higher risk in the discordant non-CBG group was close to statistical significance (Table 4).

Similarly, concordant GCB and non-GCB groups and the discordant non-GCB group had significantly lower OS (38\%, 49\% and 63\% at 5 years, respectively) than the discordant GCB, non-infiltrated GCB and non-GCB groups $(76 \%, 81 \%$ and $75 \%$, respectively, $(p<0.05)$ (Figure 3B), although the association was not independent of the R-IPI in the multivariate analysis (Table 4). 


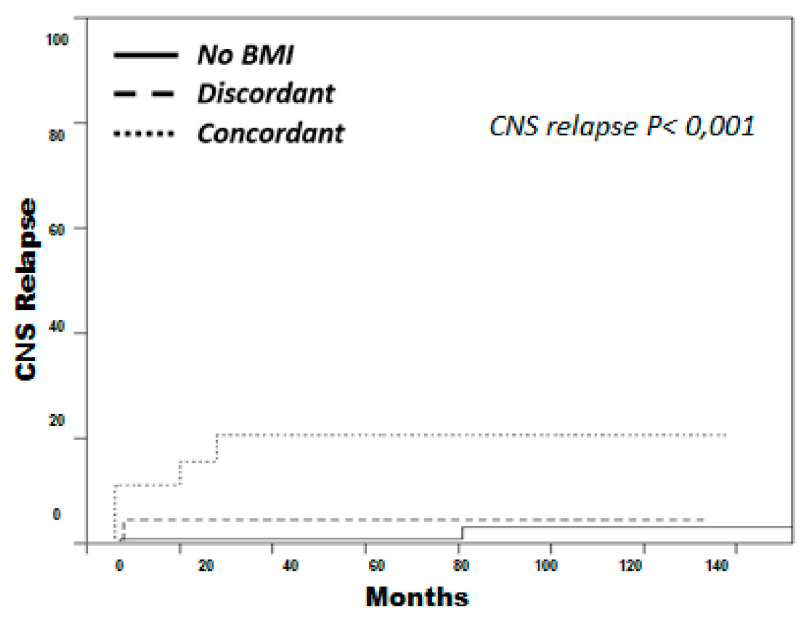

Figure 2. Cumulative incidence of central nervous system (CNS) relapse by bone-marrow infiltration type. Considering death as a potential competing risk, cumulative incidence of CNS relapse was significantly higher in the concordant group (dotted line) as compared to the discordant group (discontinuous line) and the non-infiltrated group (continuous line).
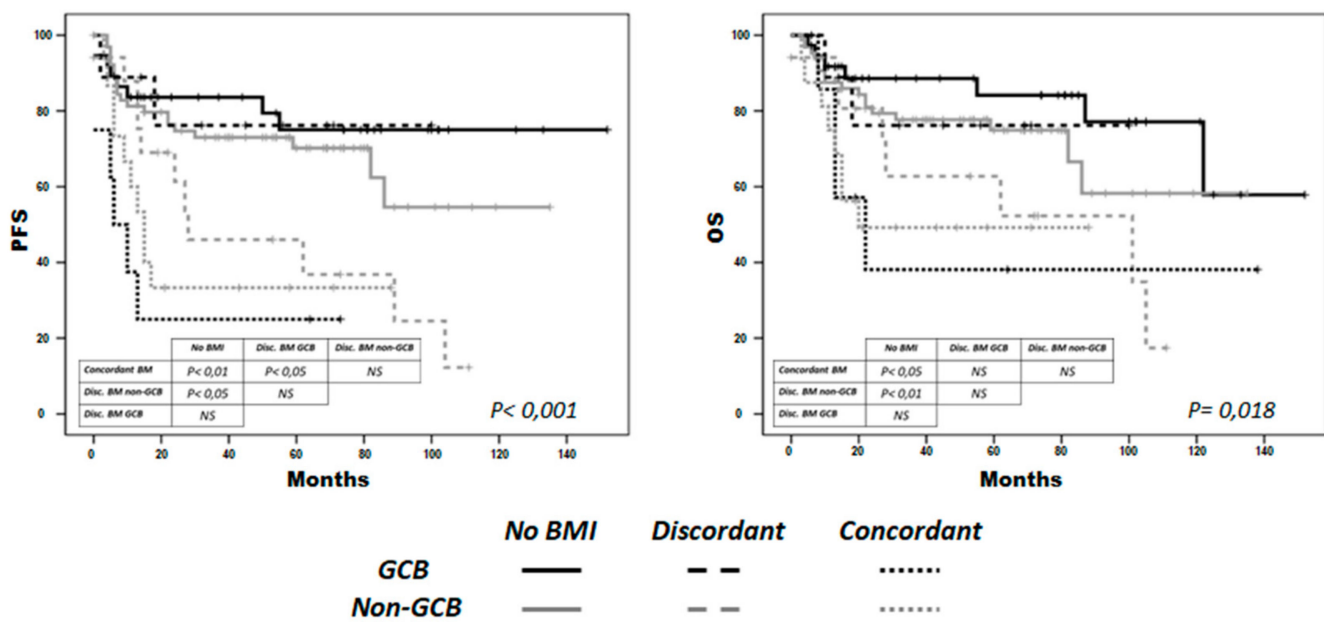

Discordant

Concordant

Non-GCB

(A)

(B)

Figure 3. Progression-free survival (PFS) (A) and overall survival (OS) (B) by bone-marrow infiltration type and cell of origin. Concordant BMI was represented as a dotted line, discordant BMI as a discontinuous line, and the non-infiltrated BMI as continuous line. Black lines represent germinal center B-cell like (GCB), while gray non-GCB DLBCL. (A) PFS was significantly decreased in both concordant BMI groups as compared to both non-infiltrated groups, and discordant GCB group $(p<0.05$, all comparisons). PFS was also lower in the discordant non-GCB group than in the non-infiltrated groups $(p<0.05)$. (B) OS was significantly lower in both concordant groups, and in the discordant non-CGB group, compared with the non-infiltrated groups $(p<0.05)$.

\section{Discussion}

The use of highly effective rituximab-containing primary therapy in DLBCL makes it more difficult to salvage patients who are refractory or who relapse [17]. Therefore, early recognition of poor prognostic patients is very important for exploring alternative first-line treatment strategies. In our series of 189 patients treated with R-CHOP-like, we observed that high-grade BM involvement had a significantly unfavorable effect on PFS, independent of R-IPI, even considering only stage IV patients. This finding is in accordance with the results reported by Sehn et al. [2] from larger series and, in agreement with these observations, a recent study published by Yao et al. [18] has suggested 
that patients with DLBCL and concordant BM involvement should be considered a distinct entity due to a more aggressive course and poor prognosis in terms of both PFS and OS. However, in our series, concordant involvement had no independent prognostic influence on OS in the multivariate analysis, probably due to the small number of patients with concordant infiltration and to the relatively high efficacy of the salvage therapies employed, including autologous and allogeneic stem cell transplantation.

Another relevant finding was the high incidence of CNS relapse in the concordant BM patients in our series. BM involvement has been cited as a risk factor for CNS involvement [8], although a study performed in the rituximab era found that BM involvement was associated with a higher rate of CNS relapse only in the presence of increased LDH [7]. In our study, concordant, but not discordant, BM involvement was a risk factor for CNS relapse, independent of CNS-IPI. This finding has been previously described by Sehn et al. in another retrospective analysis [2]. In both studies, the number of events was small and the multivariate analysis was limited to the IPI or CNS-IPI score, so larger studies are needed to confirm these results. In the recent and previously mentioned analysis from Yao et al. [18], investigators do not describe a higher incidence of CNS relapse in patients with concordant BM infiltration. However, CNS prophylaxis, including systemic high dose methotrexate (MTX) and cytarabine or intrathecal MTX, were administered to $61.8 \%$ of patients. In our series, CNS prophylaxis was administered only to $26 \%$ of patients with concordant BM infiltration, and it was only based on intrathecal triple therapy (MTX, cytarabine and hydrocortisone).

Therefore, although based upon retrospective data, as it is the case of the majority of studies that analyze the risk factors for CNS relapse [19], our results suggest that patients with concordant BM involvement are candidates for CNS evaluation and systemic CNS prophylaxis.

We explored the biological characteristics of patients with discordant BM infiltration in order to understand better their unclear prognosis. As Brudno et al. noted in their review [5], there is a paucity of published information about the biology, diagnosis, treatment and outcome of discordant lymphomas. First of all, we observed that although COO is not related to the type of infiltration, it gains relevance as a potential prognostic factor when combined with the type of BM involvement. Patients who have both discordant BM and non-GCB-DLBCL have a significantly lower PFS, the association being close to statistical significance in the multivariate analysis. To our knowledge, this finding has not been previously reported. In contrast, discordant BM in patients with GCB-DLBCL had similar outcomes to those without BM infiltration in our series of patients, which questions whether these cases should be considered stage IV. This might explain the lack of consensus about discordant DLBCL outcomes in previous studies. However, due to the small sample size of subgroups, these findings should be interpreted with caution, and should be confirmed in larger series and, preferably, with COO determination based on gene expression profile methods.

So far, FCM has not demonstrated clinical value in determining the prognosis of patients with DLBCL, but it is helpful in the phenotypic characterization Thus, in accordance with previous studies [9], we observed that FCM helps both to define the type of low-grade lymphoma observed in the BM, and to enhance the sensitivity of histology to detect BM involvement. Nowadays, when bicrestal biopsies are not commonly performed, FCM might be useful in addition to bone marrow trephine in detecting BM involvement [20]. In our series, FCM was beneficial mainly for characterizing unspecific patterns of infiltration for which there is no conclusive evidence of lymphoma, but also aided the phenotypic description of discordant cases. With the new techniques of high-sensitivity FCM and the progressive standardization of protocols [21], the detection of BM infiltration in patients with lymphoma is likely to improve, so prospective studies incorporating these new techniques are needed to assess the real clinical value of FCM in DLBCL patients.

Several studies have suggested that FDG PET can predict BM infiltration with high sensitivity in aggressive non-Hodgkin lymphomas [22,23]. However, PET is less sensitive at detecting low-grade infiltrates [24], which account for a notable percentage of the BM infiltrations in patients with DLBCL. Although identification of high-grade BM infiltration seems to be critical for determining the prognosis, 
detection of low-grade infiltrates could also have prognostic value when combined with the $\mathrm{COO}$, as mentioned before. So, the BM biopsy and aspirate might still be needed, at least in prospective studies, to better define the genuine prognostic impact of discordant involvement.

Clonality studies analyzing the relation between low-grade and high-grade lymphoma in patients with discordant BM demonstrated a very high rate of clonal identity, as previous studies have suggested [9]. These could be interpreted as potential cases of transformation from different subtypes of indolent lymphoma. Interestingly, three out of the five discordant BM cases with a CLL phenotype were not clonally related. Genomic analysis may be necessary to determine whether the mechanisms involved in transformation in this group of patients match those observed in a series of documented high-grade transformations from the different indolent lymphomas [25-28].

In our series, we found numerous genetic aberrations involving MYC, TP53, BCL2 and BCL6 in the patients with BM involvement. Particularly, we have documented a high incidence $(70 \%)$ of $(14 ; 18)$ translocations, affecting the anti-apoptotic gene $B C L 2$, in the group of patients with $B M$ infiltration and GCB-DLBCL subtype, while the frequency reported in GCB-DLBCL, irrespective of BM infiltration, is $18 \%-35 \%[29,30]$. This might explain in part the bad prognosis of this group of patients due to the clinical role of this gene in patients with DLBCL in other studies [29-32]. MYC gains were more frequently observed in the group with discordant $\mathrm{BM}$, and it was equally distributed between both COO groups. The clinical significance of $M Y C$ gains remains controversial [33], although recent reports suggest a worse clinical outcome in GCB-DLBCL [31].

TP53 deletion, and gains and translocations of $B C L 6$, were homogenously distributed in the group of patients with infiltrated BM, without any bias for any of the groups, probably due to the reduced number of patients included.

\section{Conclusions}

In conclusion, our results show that concordant BM infiltration was associated with lower PFS and a high incidence of CNS relapse independent of $\mathrm{COO}$ and IPI, suggesting that different treatments from $\mathrm{R}$-CHOP are needed for these patients. Discordant BM infiltration is clonally related to the aggressive histology in the majority of cases, and transformation mechanisms should be analyzed in future studies. In a different way to the concordant infiltration, the prognostic impact of discordant BM infiltration could be limited to the non-CGB cases. Although based on a single-center retrospective analysis with a limited number of cases, our results are intriguing, and should be confirmed in larger and prospective series. The incorporation of new, highly sensitive techniques, such as FCM and PET, as well as extensive genomic studies, will contribute in the future to the better diagnosis, characterization and prognostic evaluation of BM infiltration in patients with DLBCL.

Supplementary Materials: The following are available online at http://www.mdpi.com/2072-6694/12/2/474/s1, Figure S1: Progression-free survival (PFS) (A) and overall survival (OS) (B) by bone-marrow infiltration type considering those patients with stage IV.

Author Contributions: Conceptualization, S.A.-Á., M.A. and A.M.; Data curation, S.A.-Á. and M.A.; Formal analysis, S.A.-Á., M.A., M.G.-Á. and A.M.; Funding acquisition, M.A., M.D.C., M.G. and A.M.; Investigation, M.G.-Á., O.B., M.R., M.B., J.C.C., J.D., M.B.V., C.E., P.A., L.G.D., P.T., M.D.C., N.C.G., M.G. and A.M.; Methodology, O.B., M.R., M.B., M.B.V., N.C.G. and A.M.; Project administration, A.M.; Writing of the original draft, S.A.-Á., M.A. and A.M.; Writing - review \& editing, M.G.-Á., O.B., M.R., M.B., J.C.C., J.D., M.B.V., C.E., P.A., L.G.D., P.T., M.D.C., N.C.G., M.G. and A.M. All authors have read and agreed to the published version of the manuscript.

Funding: Instituto de Salud Carlos III: PI13/02644; Instituto de Salud Carlos III: PI15/01393; Instituto de Salud Carlos III: RD12/0036/0069; Instituto de Salud Carlos III: CB16/12/00233; Consejería de Sanidad de Castilla y León: GRS 850/A/13; Consejería de Sanidad de Castilla y León: GRS 1180/A/15; Consejería de Sanidad de Castilla y León: GRS 1350/A/16; Consejería de Sanidad de Castilla y León: GRS 1846/A/18; Consejería de Sanidad de Castilla y León: GRS 2035/A/19; Consejería de Sanidad de Castilla y León: BIO/SA78/15; Gilead Sciences: GLD17/00334.

Acknowledgments: This work was partially supported by the Instituto de Salud Carlos III (ISCIII), Spanish Ministry of Economy and Competitiveness PI13/02644, PI15/01393, RD12/0036/0069, CIBERONC-CB16/12/00233, and "Una manera de hacer Europa" (Innocampus; CEI-2010-1-0010)", the Health Council of the Junta de Castilla y León (GRS 850/A/13, GRS 1180/A/15, GRS 1350/A/16, GRS 1846/A/18, GRS 2035/A/19, and BIO/SA78/15), and 
Gilead Sciences (GLD17/00334). All Spanish funding is co-sponsored by the European Union FEDER program. The authors wish to thank Gloria Ercilla, Isabel Martín, María Teresa González (Flow Cytometry Unit), María del Pozo (Cytogenetics Unit) for their technical support, and Phil Mason for checking the English grammar of the manuscript.

Conflicts of Interest: The authors declare no conflict of interest. The authors reports grants PI13/02644, PI15/01393, RD12/0036/0069, CIBERONC-CB16/12/00233, GRS 850/A/13, GRS 1180/A/15, GRS 1350/A/16, BIO/SA78/15, GLD17/00334, GRS 1846/A/18, GRS 2035/A/19, and CB16/12/00233 during the conduct of the study, and from Gilead Sciences (GLD15/00348), Janssen, and Amgen outside the submitted work. MGA is supported by the Spanish government (FEHH). Consulting fees and/or non-financial support were reported from Abbvie (MA, MG), Amgen (MDC), BMS (MDC), Celgene (MDC, AM), Gilead (MDC, MG, AM), iQone Healthcare Export (AM), Janssen (MA, NG, MDC, MG, AM), Kyowa Kirin (AM), Kite (MDC), MSD (MDC), Novartis (SAA, MDC), Roche (MA, MDC, MG, AM), Servier (AM), and Takeda (MDC). The remaining authors have no relevant conflicts of interest to disclose.

\section{References}

1. Chigrinova, E.; Mian, M.; Scandurra, M.; Greiner, T.C.; Chan, W.C.; Vose, J.M.; Inghirami, G.; Chiappella, A.; Baldini, L.; Ponzoni, M.; et al. Diffuse large B-cell lymphoma with concordant bone marrow involvement has peculiar genomic profile and poor clinical outcome. Hematol. Oncol. 2011, 29, 38-41. [CrossRef] [PubMed]

2. Sehn, L.H.; Scott, D.W.; Chhanabhai, M.; Berry, B.; Ruskova, A.; Berkahn, L.; Connors, J.M.; Gascoyne, R.D. Impact of concordant and discordant bone marrow involvement on outcome in diffuse large B-cell lymphoma treated with R-CHOP. J. Clin. Oncol. 2011, 29, 1452-1457. [CrossRef] [PubMed]

3. Shim, H.; Oh, J.I.; Park, S.H.; Jang, S.; Park, C.J.; Huh, J.; Suh, C.; Chi, H.S. Prognostic impact of concordant and discordant cytomorphology of bone marrow involvement in patients with diffuse, large, B-cell lymphoma treated with R-CHOP. J. Clin. Pathol. 2013, 66, 420-425. [CrossRef] [PubMed]

4. Park, M.J.; Park, S.H.; Park, P.W.; Seo, Y.H.; Kim, K.H.; Seo, J.Y.; Jeong, J.H.; Kim, M.J.; Ahn, J.Y.; Hong, J. Prognostic impact of concordant and discordant bone marrow involvement and cell-of-origin in Korean patients with diffuse large B-cell lymphoma treated with R-CHOP. J. Clin. Pathol. 2015, 68, 733-738. [CrossRef]

5. Brudno, J.; Tadmor, T.; Pittaluga, S.; Nicolae, A.; Polliack, A.; Dunleavy, K. Discordant bone marrow involvement in non-Hodgkin lymphoma. Blood 2016, 127, 965-970. [CrossRef]

6. Song, M.K.; Chung, J.S.; Lee, J.J.; Yang, D.H.; Kim, I.S.; Shin, D.H.; Shin, H.J. High Ki-67 expression in involved bone marrow predicts worse clinical outcome in diffuse large B cell lymphoma patients treated with R-CHOP therapy. Int. J. Hematol. 2015, 101, 140-147. [CrossRef]

7. Lee, K.W.; Yi, J.; Choi, I.S.; Kim, J.H.; Bang, S.M.; Kim, D.W.; Im, S.A.; Kim, T.Y.; Yoon, S.S.; Lee, J.S.; et al. Risk factors for poor treatment outcome and central nervous system relapse in diffuse large B-cell lymphoma with bone marrow involvement. Ann. Hematol. 2009, 88, 829-838. [CrossRef]

8. Tomita, N.; Takasaki, H.; Ishiyama, Y.; Kishimoto, K.; Ishibashi, D.; Koyama, S.; Ishii, Y.; Takahashi, H.; Numata, A.; Watanabe, R.; et al. Intrathecal methotrexate prophylaxis and central nervous system relapse in patients with diffuse large B-cell lymphoma following rituximab plus cyclophosphamide, doxorubicin, vincristine and prednisone. Leuk Lymphoma 2015, 56, 725-729. [CrossRef]

9. Kremer, M.; Spitzer, M.; Mandl-Weber, S.; Stecker, K.; Schmidt, B.; Höfler, H.; Quintanilla-Martinez, L.; Fend, F. Discordant bone marrow involvement in diffuse large B-cell lymphoma: Comparative molecular analysis reveals a heterogeneous group of disorders. Lab Investig. 2003, 83, 107-114. [CrossRef]

10. Fisher, D.E.; Jacobson, J.O.; Ault, K.A.; Harris, N.L. Diffuse large cell lymphoma with discordant bone marrow histology. Clinical features and biological implications. Cancer 1989, 64, 1879-1887. [CrossRef]

11. Hans, C.P.; Weisenburger, D.D.; Greiner, T.C.; Gascoyne, R.D.; Delabie, J.; Ott, G.; Muller-Hermelink, H.K.; Campo, E.; Braziel, R.M.; Jaffe, E.S.; et al. Confirmation of the molecular classification of diffuse large B-cell lymphoma by immunohistochemistry using a tissue microarray. Blood 2004, 103, 275-282. [CrossRef] [PubMed]

12. Sanchez, M.L.; Almeida, J.; Vidriales, B.; Lopez-Berges, M.C.; Garcia-Marcos, M.A.; Moro, M.J.; Corrales, A.; Calmuntia, M.J.; San Miguel, J.F.; Orfao, A. Incidence of phenotypic aberrations in a series of 467 patients with B chronic lymphoproliferative disorders: Basis for the design of specific four-color stainings to be used for minimal residual disease investigation. Leukemia 2002, 16, 1460-1469. [CrossRef] [PubMed] 
13. Barrena, S.; Almeida, J.; Del Carmen García-Macias, M.; López, A.; Rasillo, A.; Sayagués, J.M.; Rivas, R.A.; Gutierrez, M.L.; Ciudad, J.; Flores, T.; et al. Flow cytometry immunophenotyping of fine-needle aspiration specimens: Utility in the diagnosis and classification of non-Hodgkin lymphomas. Histopathology 2011, 58, 906-918. [CrossRef]

14. Van Dongen, J.J.M.; Langerak, A.W.; Brüggemann, M.; Evans, P.A.S.; Hummel, M.; Lavender, F.L.; Delabesse, E.; Davi, F.; Schuuring, E.; Garcia-Sanz, R.; et al. Design and standardization of PCR primers and protocols for detection of clonal immunoglobulin and T-cell receptor gene recombinations in suspect lymphoproliferations: Report of the BIOMED-2 Concerted Action BMH4-CT98-3936. Leukemia 2003, 17, 2257-2317. [CrossRef] [PubMed]

15. Sehn, L.H.; Berry, B.; Chhanabhai, M.; Fitzgerald, C.; Gill, K.; Hoskins, P.; Klasa, R.; Savage, K.J.; Shenkier, T.; Sutherland, J.; et al. The revised International Prognostic Index (R-IPI) is a better predictor of outcome than the standard IPI for patients with diffuse large B-cell lymphoma treated with R-CHOP. Blood 2007, 109, 1857-1861. [CrossRef] [PubMed]

16. Schmitz, N.; Zeynalova, S.; Nickelsen, M.; Kansara, R.; Villa, D.; Sehn, L.H.; Glass, B.; Scott, D.W.; Gascoyne, R.D.; Connors, J.M.; et al. CNS International Prognostic Index: A Risk Model for CNS Relapse in Patients With Diffuse Large B-Cell Lymphoma Treated With R-CHOP. J. Clin. Oncol. 2016, 34, 3150-3156. [CrossRef]

17. Martín, A.; Conde, E.; Arnan, M.; Canales, M.A.; Deben, G.; Sancho, J.M.; Andreu, R.; Salar, A.; Garcia-Sanchez, P.; Vazquez, L.; et al. R-ESHAP as salvage therapy for patients with relapsed or refractory diffuse large B-cell lymphoma: The influence of prior exposure to rituximab on outcome. A GEL/TAMO study. Haematologica 2008, 93, 1829-1836. [CrossRef]

18. Yao, Z.; Deng, L.; Xu-Monette, Z.Y.; Manyam, G.C.; Jain, P.; Tzankov, A.; Visco, C.; Bhagat, G.; Wang, J.; Dybkaer, K.; et al. Concordant bone marrow involvement of diffuse large B-cell lymphoma represents a distinct clinical and biological entity in the era of immunotherapy. Leukemia 2018, 32, 353-363. [CrossRef]

19. Peñalver, F.J.; Sancho, J.M.; de la Fuente, A.; Olave, M.T.; Martín, A.; Panizo, C.; Perez, E.; Salar, A.; Orfao, A. Guidelines for diagnosis, prevention and management of central nervous system involvement in diffuse large B-cell lymphoma patients by the Spanish Lymphoma Group (GELTAMO). Haematologica 2017, 102, 235-245. [CrossRef]

20. Cabezas-Quintario, M.A.; Gomez, P.; Yuste-Del Pozo, V.; Valencia-Mesa, A.L.; Sosa, G.; Ricard, P.; Hijas-Gomez, A.I.; Pinedo, F.; Arguelles, M. Bone marrow trephine biopsy involvement by lymphoma: Pattern of involvement and concordance with flow cytometry, in 10 years from a single institution. Clin. Transl. Oncol. 2015, 18, 537-540. [CrossRef]

21. Van Dongen, J.J.M.; Lhermitte, L.; Böttcher, S.; Almeida, J.; Van der Velden, V.H.J.; Flores-Montero, J.; Rawstron, A.; Asnafi, V.; Lecrevisse, Q.; Lucio, P.; et al. EuroFlow antibody panels for standardized n-dimensional flow cytometric immunophenotyping of normal, reactive and malignant leukocytes. Leukemia 2012, 26, 1908-1975. [CrossRef] [PubMed]

22. Cortés-Romera, M.; Sabaté-Llobera, A.; Mercadal-Vilchez, S.; Climent-Esteller, F.; Serrano-Maestro, A.; Gámez-Cenzano, C.; González-Barca, E. Bone marrow evaluation in initial staging of lymphoma: 18F-FDG PET/CT versus bone marrow biopsy. Clin. Nucl. Med. 2014, 39, e46-e52. [CrossRef] [PubMed]

23. Khan, A.B.; Barrington, S.F.; Mikhaeel, N.G.; Hunt, A.A.; Cameron, L.; Morris, T.; Carr, R. PET-CT staging of DLBCL accurately identifies and provides new insight into the clinical significance of bone marrow involvement. Blood 2013, 122, 61-67. [CrossRef]

24. Jerusalem, G.; Beguin, Y.; Najjar, F.; Hustinx, R.; Fassotte, M.F.; Rigo, P.; Fillet, G. Positron emission tomography (PET) with 18F-fluorodeoxyglucose (18F-FDG) for the staging of low-grade non-Hodgkin's lymphoma (NHL). Ann. Oncol. 2001, 12, 825-830. [CrossRef]

25. Chigrinova, E.; Rinaldi, A.; Kwee, I.; Rossi, D.; Rancoita, P.M.; Strefford, J.C.; Oscier, D.; Stamatopoulos, K.; Papadaki, T.; Berger, F.; et al. Two main genetic pathways lead to the transformation of chronic lymphocytic leukemia to Richter syndrome. Blood 2013, 122, 2673-2682. [CrossRef] [PubMed]

26. Okosun, J.; Bödör, C.; Wang, J.; Araf, S.; Yang, C.Y.; Pan, C.; Boller, S.; Cittaro, D.; Bozek, M.; Iqbal, S.; et al. Integrated genomic analysis identifies recurrent mutations and evolution patterns driving the initiation and progression of follicular lymphoma. Nat. Genet. 2014, 46, 176-181. [CrossRef] 
27. Pasqualucci, L.; Khiabanian, H.; Fangazio, M.; Vasishtha, M.; Messina, M.; Holmes, A.B.; Ouillette, P.; Trifonov, V.; Rossi, D.; Tabbo, F.; et al. Genetics of follicular lymphoma transformation. Cell Rep. 2014, 6, 130-140. [CrossRef]

28. Jiménez, C.; Alonso-Álvarez, S.; Alcoceba, M.; Ordóñez, G.R.; García-Álvarez, M.; Prieto-Conde, M.I.; Chillón, M.C.; Balanzategui, A.; Corral, R.; Marín, L.A.; et al. From Waldenström's macroglobulinemia to aggressive diffuse large B-cell lymphoma: A whole-exome analysis of abnormalities leading to transformation. Blood Cancer J. 2017, 7, e591. [CrossRef]

29. Iqbal, J.; Sanger, W.G.; Horsman, D.E.; Rosenwald, A.; Pickering, D.L.; Dave, B.; Dave, S.; Xiao, L.; Cao, K.; Zhu, Q.; et al. BCL2 translocation defines a unique tumor subset within the germinal center B-cell-like diffuse large B-cell lymphoma. Am. J. Pathol. 2004, 165, 159-166. [CrossRef]

30. Visco, C.; Tzankov, A.; Xu-Monette, Z.Y.; Miranda, R.N.; Tai, Y.C.; Li, Y.; Liu, W.M.; d'Amore, E.S.; Li, Y.; Montes-Moreno, S.; et al. Patients with diffuse large B-cell lymphoma of germinal center origin with BCL2 translocations have poor outcome, irrespective of MYC status: A report from an International DLBCL rituximab-CHOP Consortium Program Study. Haematologica 2013, 98, 255-263. [CrossRef]

31. Ennishi, D.; Mottok, A.; Ben-Neriah, S.; Shulha, H.P.; Farinha, P.; Chan, F.C.; Meissner, B.; Boyle, M.; Hother, C.; Kridel, R.; et al. Genetic profiling of MYC and BCL2 in diffuse large B-cell lymphoma determines cell of origin-specific clinical impact. Blood 2017, 129, 2760-2770. [CrossRef] [PubMed]

32. Chapuy, B.; Stewart, C.; Dunford, A.J.; Kim, J.; Kamburov, A.; Redd, R.A.; Lawrence, M.S.; Roemer, M.G.M.; Li, A.J.; Ziepert, M.; et al. Molecular subtypes of diffuse large B cell lymphoma are associated with distinct pathogenic mechanisms and outcomes. Nat. Med. 2018, 24, 679-690. [CrossRef] [PubMed]

33. Testoni, M.; Kwee, I.; Greiner, T.C.; Montes-Moreno, S.; Vose, J.; Chan, W.C.; Chiappella, A.; Baldini, L.; Ferreri, A.J.; Gaidano, G.; et al. Gains of MYC locus and outcome in patients with diffuse large B-cell lymphoma treated with R-CHOP. Br. J. Haematol. 2011, 155, 274-277. [CrossRef] [PubMed]

(C) 2020 by the authors. Licensee MDPI, Basel, Switzerland. This article is an open access article distributed under the terms and conditions of the Creative Commons Attribution (CC BY) license (http://creativecommons.org/licenses/by/4.0/). 